Supporting Service for the Mentally Handicapped

E. B. McDowal., M.R.C.PSYCH. . . . . . . 481

Social Workers and Family Doctors

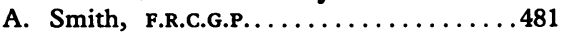

Localization of Hepatitis $B$ Antigen in Liver Organ Cultures

A. J. Zuckerman, M.D., and R. G. Bird,

PH.D., D.T.M.\&H. . ..............482

Severely Malformed Children

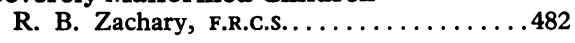

Multiple Factors in Leukaemogenesis

P. G. Smith, and others...........482

Redesign of Medical Records in General Practice

J. M. Brown, M.R.C.G.P.; D. Jarratt, M.B.;

H. C. I. Bywater, M.B., and others. . . ...483

Drug Data Sheets

R. E. Tringham . . . . . . . . . . 484

Coeliac Disease in the West of Ireland

E. J. Moynahan, F.R.C.P.; P. Asquith, M.D.,

and W. T. Cooke, F.R.C.P..........484

Medical Staff Dining-rooms

R. C. Redman, M.B., B.CH..........485
Actinomycin D for Wilms's Tumour

R. D. T. Jenkin, M.B.; T. F. Sandeman,

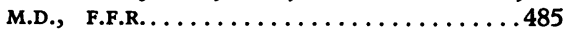

Efficacy of Whooping-cough Vaccines

N. W. Preston, F.R.C.PATH............485

Discrimination against Rhodesian Nurses

P. L. C. Diggory, F.R.C.o.G. . . . . . . . . . 486

Resources and Needs in Africa

G. W. St. J. Chadwick. . . . . . . . . . . 486

Prescribing Mandrax

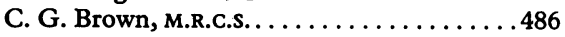

Behçet's Syndrome and Oral Fibrinolytic

Therapy

W. J. Cuncliffe, M.D., and others. . . . . . . 486

Toxicity of Benorylate

V. Wright, F.R.C.P., and I. Haslock, M.D. . . . 487

Eclampsia and Social Change in the Tropics

D. Jenkinson, M.B..............487

Diagnosis of Multiple Pregnancy

P. A. Barker, M.B., and D. V. Cashman,

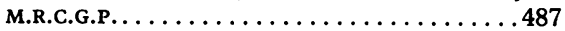

Nitrazepam and the Subconscious

T. Goossens, M.D., and others........488
Discriminant Value of Thyroid Function

Tests

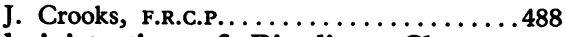

Administration of Disodium Chromo-

glycate to Young Children

H. E. Williams, F.R.A.C.P., and P. D. Phelan,

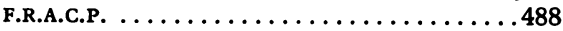

Purpura Associated with Vomiting in

Pregnancy

M. F. Burke, F.R.C.s., and Janet M. Marks,

D.M. ...........................488

Grades of Hypothyroidism

R. F. Harvey, M.D. . . . . . . . . . . . . .488

Mediterranean Anaemia in Antiquity

W. I. Menke, M.D. . . . . . . . . . . . . . 489

Hysterectomization etc.

J. Mantle. . . . . . . . . . . . . . . . . . . . .4899

Distinction Awards

G. I. B. Da Costa, F.R.C.S.ED. . . . . . . . . . . . 489

Consultants' Superannuation

P. F. Plumley, F.R.C.s.............. 489

Representation of Junior Members

M. G. F. Crowe, M.R.c.G.P.. . . . . . . . . 490

Reorganization of the B.M.A.

M. E. M. Cook, M.R.C.G.P., and others. . . . .490 G.P.s' Terms of Service

A. F. Oakley, м.B................490

\section{Supporting Service for the Mentally Handicapped}

SIR,-In a recent article (24 February, p. 435) you outlined an apparently critical medical situation in the field of mental subnormality, and as a corollary you asked whether teachers, psychologists, and social workers should be invited to fill the gap. But neither your article nor the subsequent correspondence pinpointed the present fragmented administration as the single element in the care of the mentally handicapped that has prevented, is preventing, and will continue to prevent the emergence of a satisfactory comprehensive service.

The care of mental subnormality is unique in that we are not dealing with illness but with social incompetence. The management is very largely social and educational; but the medical background is clearly crucial in pre vention, in diagnosis and clinical treatment in assessment of progress, and in the psychiatric handling and therapy of behaviour disturbance. It is, I suppose, simply because it is primarily neither social nor medical nor educational but is a discipline sui generis that the present administrative situation has evolved by piecemeal legislation. It is split down the middle by the hospital/community dichotomy; further subdivided by the separate social, medical, and educational responsibilities in local government; complicated by the fact that hostels may be staffed, trained, and managed by voluntary bodies as well; bedevilled by varying pay structures and career prospects in similar services; and, lacking any co-ordinating direction whatever, it is knit together only by the selfless, skilled work of the many who devote their lives to the care of the mentally handicapped.

No navy could be an effective force with- out a central command, however good the different ships might be; and the analogy is a fair one in this context to emphasize how necessary it is to introduce a fundamentally new administrative system. The care of a mentally handicapped member of society should clearly be the responsibility of single body, so that his diverse needs can be unified within the compass of an entirely new national authority, which would be accountable for the administration, planning, and operation of the whole service. Such a body would co-ordinate and align the policies and practice of all aspects of the work. It should be fully funded to undertake social care, hospital treatment, and all ancillary services and would maintain a close, defined liaison in educational matters. In 1972 a proposal for such a department was introduced for debate in the House of Lords by Lord Davidson, and elsewhere the need for such a national administrative authority has been considered. But as yet no further progress appears to have been made.

Signs of inadequacy and symptoms of crisis are constantly recurring, and they painfully illustrate the need for a properly coordinated administration. The suggested service would pose many problems and require detailed preparation, and the legislation would perhaps add further complication to an era which is already beset by great changes. Nevertheless there are no overwhelming difficulties in formulating an outline scheme for the work of such a new department, and it is to be hoped that those in authority will be able to consider the proposal in principle, and perhaps initiate a feasibility study. The national press and the public could perhaps add their support to a project which, in its limited field, would constitute a major progressive reform.-I am, etc.,

Brockenhurst, Hants

E. B. MCDowaLL

\section{Social Workers and Family Doctors}

SIR,-Drs. D. G. Craig and P. J. Travis (28 April, p. 243) end their letter by asking if other doctors agree with them that it is time family doctors experimented with the employment of social workers. I do.

Two years ago the lecturer in social work at this university asked me if I would take a social work student for a fortnight over the Christmas holidays. He explained that this was the only time in her curriculum which could be spared and that she had volunteered to give up her Christmas holidays to do it as an elective period. She was a great success. My partners and I handed over the social work problems we happened to be dealing with at the time, and she showed what could be done by an intelligent girl who was in her element dealing with people. She illuminated family situations, identified and set out to solve problems, and surprised everybody by being willing to cope with crises at any time. Moreover, she continued to follow up patients for some time after her attachment was over.

An exceptional girl no doubt, but not, it seems, unique. In Social Work in General Practice $^{1}$ the whole-time attachment of a social worker to a group practice in Camden is described. She also found satisfaction in taking on all comers at any time, was willing 
to intervene immediately in crisis situations, and worked out a shorter and more practical technique of interviewing than social workers are traditionally taught. I suspect that many more social workers would do the same if given the chance. At present most of them can only be contacted during office hours and then only if the clerk in the social services department office chooses to tell them that you phoned. The ideal would be local-authority attachment to particular practices, but the next best thing would be reimbursement of the social worker's salary. -I am, etc.,

ANDREW SMITH Newcastle upon Tyne Lecturer in Family Medicine,
University of Newcastle upon Tyne

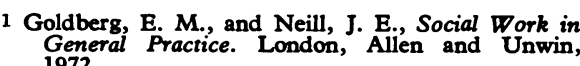
Gener
1972.

\section{Localization of Hepatitis B Antigen} in Liver Organ Cultures

SIR,-Hepatitis B antigen has been produced in organ cultures of human embryo liver inoculated with a limited number of known infective sera. ${ }^{1}$ One serum, referred to as G.C., was obtained from a young healthy volunteer blood donor whose blood recently caused two deaths from hepatitis in transfused recipients. Examination by electron microscopy of ultrathin sections of a liver organ culture inoculated with $0.1 \mathrm{ml}$ of this serum revealed the presence of spherical particles measuring 20-22 $\mathrm{nm}$ in diameter in both the cytoplasm and nucleus of hepatocytes (see figure). The particles were detected four and seven days after inoculation of the organ culture. These particles were present in many of the cells, principally at the rim of the culture. Such particles were not found in control organ culture preparations inoculated with normal human serum.

These particles are very similar to the hepatitis B antigen particles described in anti- gen-positive liver biopsy material by a number of other investigators. ${ }^{2-5}$ Nowoslawski et al. ${ }^{6}$ demonstrated by immunofluorescence the presence of hepatitis $B$ antigen in the cytoplasm as well as the nucleus of hepatocytes of six patients with lymphoproliferative disorders; but by electron microscopy only intranuclear particles were found. These particles were identical to those we describe now. Specific fluorescence was demonstrated in many of the hepatocytes by the direct im. munofluorescent antibody technique ${ }^{7}$ in 5-7 $\mu \mathrm{m}$ sections of the same liver organ culture preparation inoculated with serum G.C. No fluorescence was detected in the control organ cultures.-We are, etc.,

\section{A. J. ZuCKerman}

R. G. BIRD

Hepatitis Research Unit

London School of Hygiene and Tropical Medicine,

1 Zuckerman, A. J., Baines, P. M., and Almeida, 2 Juang., N. N., American fourmal of Pathology, 1971, 64, 483 .

Caramia, F., De Bac, C., and Ricci, G., American Fournal of Disease of Children, 1972, 123, 309. L.; and Spears, R. L., Archives of Pathology, 1972, 94, 258.

Almeida, J. D., Gioannini, P., Scalise, G., and 1973, 26, 113. .', Joumal of Clinical Pathology,

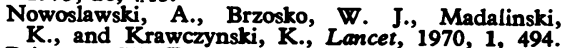
Brighton, W. W. D., Taylor, P. B., and Zuckerman A. J., Nature New Biology, 1971, 232, 57.

\section{Severely Malformed Children}

SIR,-In the tape-recorded discussion on malformed children (5 May, p. 284) Mr. H. B. Eckstein draws attention to a strange anomaly-namely, that untreated babies in first-class centres of baby care have all died "within a month," "within eight months," or similar figures, and yet surgeons are seeing a number of untreated cases for salvage from ther hospitals.

The " $100 \%$ success" rate for the notreatment policy contrasts with my own

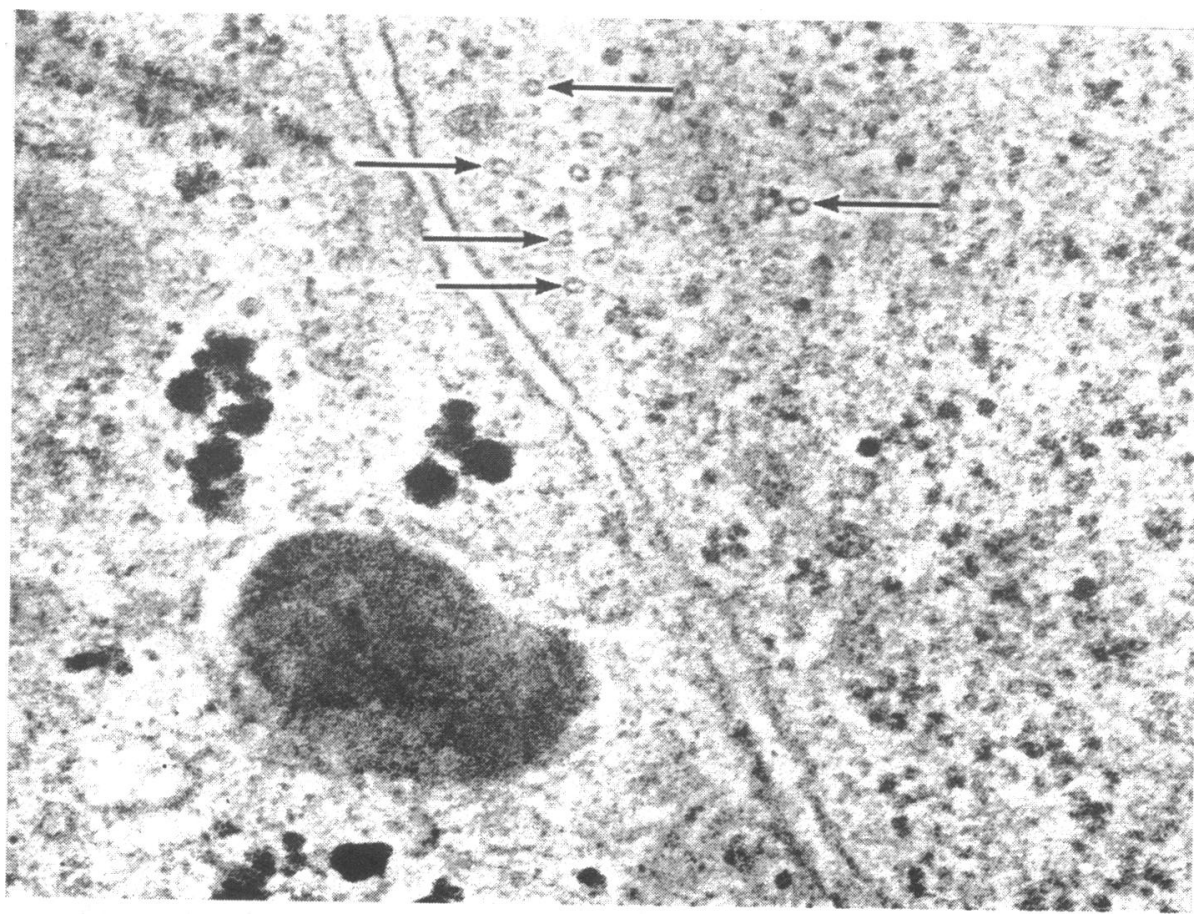

Virus-like particles of hepatitis $B$ antigen in the cytoplasm of a hepatocyte from an inoculated human embryo liver organ culture at day $7 \times 100,000$ personal experience 25 years ago, when no patients received primary surgery and most of them received ordinary home care. The survival of quite a number of the latter makes one wonder whether the ordinary standards of baby care are worse than 25 years agoor is there some other factor in their management?

Another impression which comes over in the recording-perhaps wrongly-is the apparent lack of concern for the maximum fulfilment and the happiness of these severely affected children during their life span, however long or short that may be. One is particularly concerned that the psychiatrist in the discussion does not even hint at the feelings of contentment and security which at this age are important aspects of happiness for the child and which come from knowing and being loved by one person (usually the mother).

A severely disabled child needs this personal affection and attachment, perhaps even more than an able-bodied one and if this poses a heavy burden in the mother and the family, I should have thought that the psychiatrist would be the very one to propose support for the family rather than elimination of the child from the family environment.-I am, etc.,

R. B. ZACHARY Sheffield

\section{Multiple Factors in Leukaemogenesis}

SIR,-In their study of children with leukaemia Bross and Natarajan ${ }^{1}$ investigated the association between irradiation in utero and some "indicators of susceptibility" (viral infection, bacterial infection, and allergy) shown by the leukaemic child from birth up to a time six months before diagnosis. They and you, in a leading article (21 October, 1972 , p. 128), interpreted their results as showing that "the apparently harmful effects of antenatal irradiation are greatly increased in certain susceptible subgroups of children possessing the indicators associated with slightly higher intrinsic risk of leukaemia." However, these findings may be interpreted as showing that children with leukaemia are simply more prone to viral and bacterial infections and allergies before clinical onset of the disease (or are more likely to report such conditions in a retrospective study) and that irradiation is a red herring in this argument. If this is correct, the relative risk of irradiation (that is, the ratio of the risk of a child irradiated in utero developing leukaemia to the risk of a child not so irradiated developing leukaemia) would be the same in children reporting such conditions as those not so doing.

Bross and Natarajan kindly supplied us with the data on their leukaemic patients and controls tabulated by age at diagnosis, intrauterine radiation history, and susceptibility indicators of infections or allergies (tables and II). Table III shows the relative risks of irradiation in each age group for those reporting viral or bacterial infections or allergies $\left(\mathbf{R}_{1}\right)$ and those not reporting such diseases $\left(\mathbf{R}_{2}\right)$. For example, in the first row of table III the relative risk of irradiation in children 1-4 years old reporting viral infections is obtained from tables I and II by a comparison of cases and controls reporting virus infections- $R_{1}=(15 \times 43) /(32 \times 12)=$ $1 \cdot 68$. Similiarly the relative risk of irradiation 